\title{
Les instruments domestiques dans la musique populaire suisse
}

\section{Brigitte Bachmann-Geiser}

Traducteur : Isabelle Schulte-Tenckhoff

\section{OpenEdition \\ Journals}

Édition électronique

URL : http://journals.openedition.org/ethnomusicologie/2340

ISSN : 2235-7688

Éditeur

ADEM - Ateliers d'ethnomusicologie

Édition imprimée

Date de publication : 1 janvier 1989

Pagination : 183-201

ISBN : 2-8257-0178-5

ISSN : 1662-372X

Référence électronique

Brigitte Bachmann-Geiser, «Les instruments domestiques dans la musique populaire suisse », Cahiers d'ethnomusicologie [En ligne], 2 | 1989, mis en ligne le 15 septembre 2011, consulté le 19 avril 2019. URL : http://journals.openedition.org/ethnomusicologie/2340 


\title{
LES INSTRUMENTS DOMESTIQUES DANS LA MUSIQUE POPULAIRE SUISSE*
}

\author{
Brigitte Bachmann-Geiser
}

Les instruments domestiques sont polyvalents en ce sens qu'il s'agit d'ustensiles quotidiens transformés pour la circonstance en des objets destinés à produire un bruit, un rythme ou - plus rarement - une mélodie. Tout comme les outils de travail qui leur sont apparentés sous ce rapport, les instruments domestiques appartiennent traditionnellement à l'instrumentarium populaire; de ce fait, ils relèvent d'un champ d'étude marginal situé aux confins de l'ethnologie européenne et de la musicologie. L'organologue les chercherait donc en vain dans le Real-Lexikon der Musikinstrumente de Curt Sachs, publié en 1913. Mais depuis peu, on les étudie conjointement avec les instruments enfantins $^{1}$ et dans le cadre d'un inventaire systématique des instruments de musique populaire européens ${ }^{2}$.

Il n'est pas difficile de trouver la raison du manque d'intérêt envers ces instruments de percussion primitifs dont la hauteur du son est souvent indéterminée et parmi lesquels on compte casseroles, couvercles, louches, bouteilles, planches à laver, manches à balai et cuillers. A la Renaissance, les traités organologiques s'adressaient aux mélomanes des couches dominantes et aux lecteurs fortunés, qui dédaignaient la musique et les instruments des pauvres. Cette attitude des élites envers la pratique musicale populaire fut documentée en 1511 par Sebastian Virdung dans son ouvrage Musica getutscht (cf. 1970).

\footnotetext{
Traduit de l'allemand par Isabelle Schulte-Tenckhoff.

Mis à part un essai peu connu de Sachs (1926), on peut dire que Herrmann (1978) est la première à se pencher sur ce nouvel objet d'étude. Pour ce qui est des instruments enfantins, divers chercheurs ont exposé leur point de vue à ce sujet lors de la troisième réunion de travail de l'International Study Group on Folk Musical Instruments de l'International Council for Traditional Music, qui se tint en 1983 à Piran en Yougoslavie. Les actes de ce congrès ont paru en 1985 à Stockholm dans le volume $N^{\circ} 8$ de Studia instrumentorum musicae popularis. Évoquons également, à ce sujet, le numéro spécial de World of Music $\mathrm{N}^{\circ} 3$, paru en 1987 sous le titre Children's music and musical instruments, où figurent des articles par S. De LorenziSchenkel, B. Bachmann-Geiser et V. Atanasov. Voir, enfin, Bachmann (1984).

2 Les volumes déjà parus du Handbuch der europäischen Volksmusikinstrumente, édités par Ernst Emsheimer et Erich Stockmann 1967), abordent aussi, en marge, les instruments domestiques. Voir Sárosi (1967), Kunz (1974), Bachmann-Geiser (1981), Elschek (1983) et Kumer (1986).
} 
Après avoir présenté, à l'aide de descriptions et de xylographies, tous les instruments de l'orchestre de la Renaissance, l'auteur en vient aux «instruments idiots" ("dorlichen [ = törichte] instrumenta»), mais la tâche de les "nommer, illustrer et décrire» ("dye zuo nennen zuo malen und zuo beschreiben») ne l'enchante guère. Parmi les instruments «idiots», auxquels l'auteur attribue le qualificatif méprisant de «göckelspiel» («jeu de bouffon»), il énumère divers instruments populaires toujours pratiqués de nos jours en Suisse, dont deux instruments domestiques.

bañoas/roelcbes ener ein barpfen batgenennet/bas beift ber ander cyuleyr/vñ

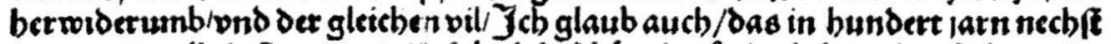
vergangenalle inftrumenta/jo fubetl//o jebón/fo git /ond fo wol geffalt getmas

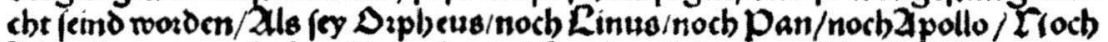
Eeitier der poeten/bab gejeben ober geborec/ vnno bas mer ift nuig licb geacbret

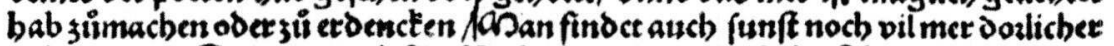

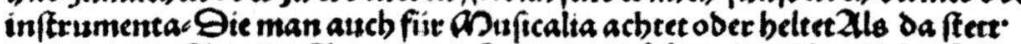

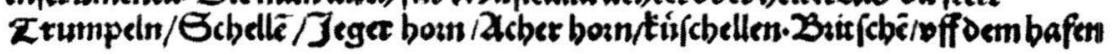

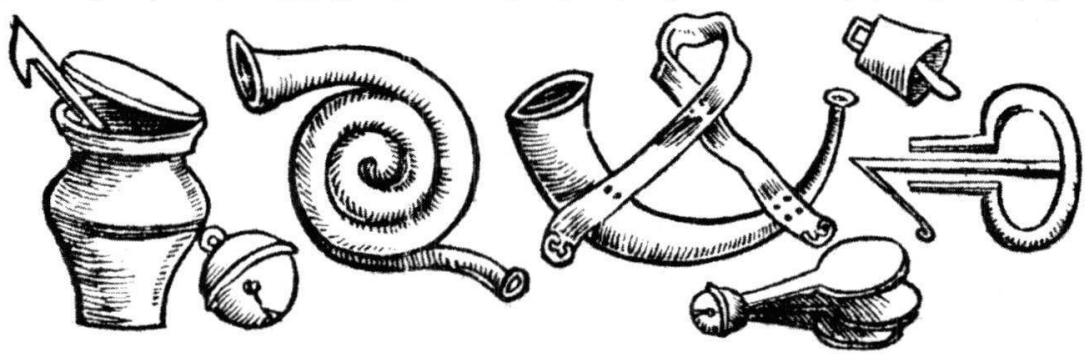

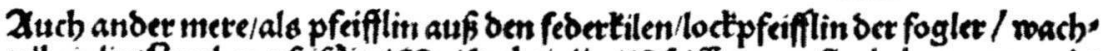
telbeinlin/Rercben $p$ ferfflin/QDaifen beinlin/p priffen von ftrobdmen gemache Pfeiffen bon den faffrigen rinden Der bóm/oon ben pletern bet b\$ $m /$ bas mä ges

Fig. 1: A gauche, «Britschen/uff dem hafen», in Sebastian Virdung, Musica getutscht, Bâle 1511.

Ainsi mentionne-t-il le «Hafenklopfen» (= «frapper sur la marmite») auquel il applique le terme de «Britschen/uff dem hafen» et qu'il assortit d'un dessin de marmite en terre cuite frappée avec une grande cuiller (fig. 1). Peu après, dans la seconde moitié du XVI ${ }^{\mathrm{e}}$ siècle, l'artiste Tobias Stimmer, de Schaffhouse, illustre le jeu du même instrument dans sa célèbre série de xylographies de musiciens, conservée actuellement au Metropolitan Museum à New York. Le poème explicatif qui l'accompagne attribue le «Hafenscharren» ("gratter la marmite») aux instruments du bouffon et rappelle ainsi le qualificatif d'«idiot» que Virdung réservait aux instruments de musique populaire.

Virdung est aussi le premier en Suisse qui décrit l'usage d'une clef pour siffler - «in dye schlüssel zu pfeiffen» -, tel qu'il est illustré par une peinture à 
l'huile exécutée vers 1540 par un artiste vraisemblablement originaire du sud de l'Allemagne ${ }^{3}$. La coutume de transformer une longue clef creuse en une flûte verticale bouchée à une extrémité existait encore dans les années trente, puisqu'elle a étonné Hanns in der Gand qui la mentionne brièvement dans son important essai sur les instruments de musique populaire suisse (1937: 99); Alfons Maissen, célèbre collectionneur et éditeur de chansons populaires romanches, s'en souvient aussi comme d'une coutume enfantine de sa jeunesse.

Ce sont maintenant les instruments domestiques recueillis au cours de nos propres recherches sur le terrain (1971-1986) qui seront décrits selon deux fonctions: en tant que contre-instruments, et en tant qu'instruments de substitution. Ensuite, il sera question du succès et de l'effet provoqués par ces instruments dans la skifflemusic suisse, en tant que signes d'un dénuement voulu, puis dans le mouvement folk et la musique populaire traditionnelle.

\section{Les contre-instruments}

Parmi les charivaris hivernaux, auxquels on attribuait la fonction de chasser les démons et d'assurer la fertilité, on compte celui de la Saint-Sylvestre scolaire (Schulsilvester) de Zurich, qui se déroule le dernier jour de classe de l'année civile. Pourtant, de nos jours, les élèves (généralement en petits groupes) ne se préoccupent plus guère de chasser les mauvais esprits. Ils s'appliquent plutôt à perturber à l'aube le sommeil paisible des citoyens, en poussant des cris et en faisant un vacarme instrumental. A côté des idiophones autochtones, tels les instruments de percussions faits de récipients en métal et divers types de grelots confectionnés à partir de boîtes de conserve, ce concert matinal comprend traditionnellement des cornes d'animaux, des cornes de brume, des sifflets à roulettes et des toupins; tous ces signaux sonores sont conservés au foyer ou empruntés pour la circonstance. Mis à part les pétards (en principe interdits), l'élément sonore dominant du Schulsilvester est encore de nos jours le bruit de deux couvercles entrechoqués, qu'ils soient en tôle, en aluminium, en émail ou, plus récemment, en acier inoxydable. Sur nos 110 informateurs, 98 ont affirmé que ces cymbales représentent l'instrument par excellence du Schulsilvester. Pour 48 d'entre eux, cet idiophone est même prépondérant dans le charivari de la ville de Zurich. Contre la promesse d'être obéissants durant la période précédant Noël, les enfants reçoivent de leur mère ces ustensiles de cuisine dont le rôle d'instrument bruyants lors de la Saint-Sylvestre zurichoise est attesté depuis un siècle. Dans son poème «Sylvester», Eduard Schönenberger écrivit en 1885 :

Ce détail figure dans le Musikbild au domaine de «Schipf» à Herrliberg $(\mathrm{ZH})$. Voir à ce sujet Jakob et Briner (1961). 
«Sie ziehnd durs Dörfli us und $i$ mit Schele, Horn und Flöte und Pfannendeckel, Gloggespiel, mit Trumme und Trumpete» ${ }^{4}$.

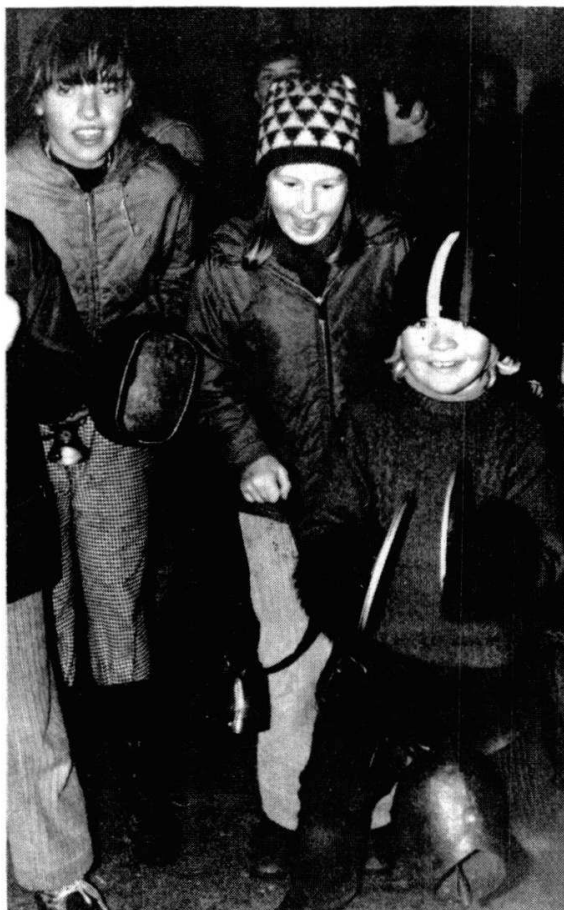

Fig. 2

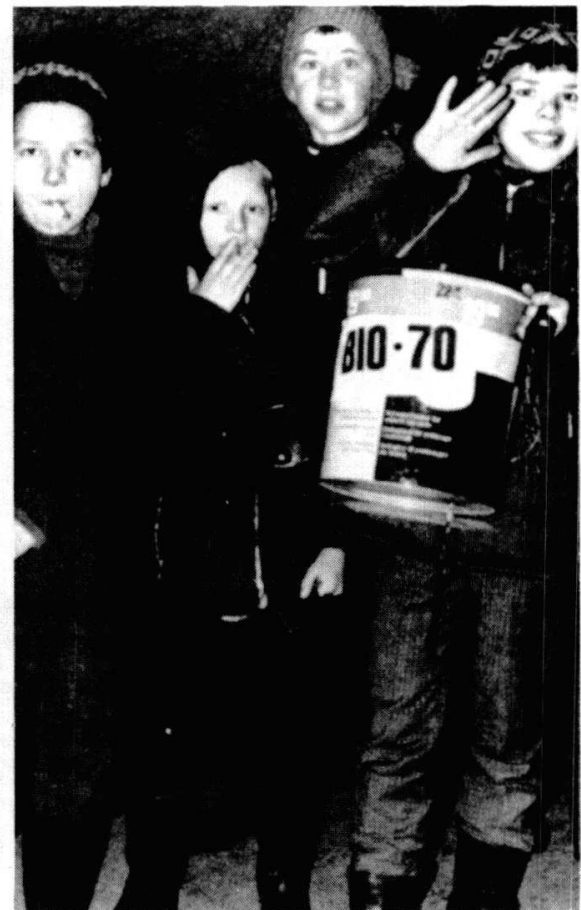

Fig. 3

Fig. 2 et 3: Schulsilvester à Herschmettlen (ZH), 1971. Photos: B. Bachmann-Geiser.

Si l'instrument national zurichois disparaît peu à peu, c'est probablement pour la raison suivante, selon un journaliste de la Neue Zürcher Zeitung:

«Peut-être les mères hésitent-elles maintenant à céder les couvercles en acier chromé de leurs casseroles en téflon» ${ }^{5}$.

Dans son poème "Schuelsilväschter i de Stadt», Barbara Egli observe la même tendance:

«Ils vont et viennent dans le village/avec toupins, cor à bouc et flûte/et couvercles, sonnailles/avec tambours et trompettes». In: Goldene Zeit: Kinderlieder und Idyllen, Zürich 1893-1897.

$5 \quad$ Le 24 decembre 1975. 
«... Und doo i de Stadt!

Ais höcklet für sich

und bimbelet mit emene

schüüche Glöggli.

E Mueter rüef abe

vom Balkon:

Daneli, gäll

heb Sorg zu de Pfanneteckel

und leg dHäntschli $a »^{6}$.

Jusqu'à une période récente, voire même encore aujourd'hui, le Schulsilvester zurichois comporte deux phases. La première se déroule à l'aube, lorsque les enfants, échappant à la surveillance des adultes, se promènent dans la ville en faisant du vacarme et des farces. Elle est suivie d'un programme récréatif souvent élaboré par les élèves eux-mêmes, qui a lieu en classe en présence de l'enseignant. Les instruments utilisés dans l'un et l'autre cas sont clairement différenciés. Dans la rue, les élèves se servent soit d'instruments qu'ils ont fabriqués eux-mêmes, soit d'instruments improvisés au moyen d'ustensiles de ménage qui sont, dans le meilleur des cas, des outils de travail ou des avertisseurs sonores puissants. Les interludes musicaux à l'école, en revanche, calqués sur le récital de concert, ne font apparaître que les instruments connus des leçons de musique. A la différence de la répétition générale des pièces de Noël, le charivari enfantin de la Saint-Sylvestre témoigne, en outre, d'une opposition nette. Ludvik Bielawski (1985: 110) a attiré l'attention sur «le refus de faire de la musique normalement», tandis que Gottfried Habenicht, dans sa monographie sur le Kastenratschen à Ebnet près de Fribourg en Brisgau, voit dans les instruments du Vendredi saint, non seulement des substituts aux cloches d'église qui viennent de se taire, mais, plus encore, une véritable antithèse.

Si l'on peut qualifier de contre-instruments les couvercles entrechoqués lors du Schulsilvester zurichois, de la Tschättermusik («musique de charivari») de Laufenburg (Argovie), du charivari matinal d'Altdorf (Uri), du charivari carnavalesque de Saignelégier (Jura) et de la Hudlete ${ }^{7}$ de Wimmis (Berne), ce nouveau concept s'applique aussi, sinon davantage, à la Saugeige.

Le terme de Saugiige ou Moregiige ${ }^{8}$ designe à Soleure, en Haute-Argovie bernoise, dans les environs d'Anêt (Berne) et à Fribourg en Brisgau, le pétrin en bois de sapin, long de deux mètres, transformé en instrument domestique. Dans nombre de ménages paysans du Mittelland, le pétrin est toujours en usage

6 «Et ici dans la ville:/Un enfant est assis, seul/et fait sonner une cloche timide./Une mère l'appelle du balcon:/Daniel, fais attention aux couvercles/et mets tes gants». In: Himmel und Höll und Hüpfistai, Zurich 1976.

7 Hudlete: terme désignant une tradition locale de Nouvel An.

8 Saugiige: «violon de cochon», Moregiige: «violon de truie». Cf. Schweizerisches Idiotikon. Wörterbuch der Schweizerdeutschen Sprache, vol. 2. Frauenfeld, 1985, colonne 151. 
pour préparer de grandes quantités de pâte à pain, car en échange de leurs céréales, les cultivateurs recevaient jusqu'à récemment, non seulement de l'argent mais aussi de la farine. En tant qu'instrument bruyant traditionnel, le pétrin repose à l'envers sur une longue planche non rabotée; souvent, les deux sont placés sur une charrette à bras. Un garçon s'assied en cavalier sur le pétrin, tandis que quatre autres commencent la «musique» en tenant le pétrin par les anses et en le frottant sur la planche en un mouvement de va-et-vient. Le vacarme ainsi provoqué se fait entendre à Soleure chaque année lors de la Chesslete 9 à l'aube du «Jeudi gras» (le jeudi précédant le Mercredi des Cendres) (fig. 4), mais on l'emploie également en tant qu'instrument de réprimande. Jakob Hofstätter a décrit l'utilisation du pétrin et d'autres instruments de bruit dans le canton de Soleure:

«Voici une coutume de mariage, qui survit dans certaines régions: si les nouveaux mariés ne plaisent pas aux jeunes gens du village, par exemple lorsque la jeune fille originaire du village décide d'épouser un étranger alors qu'un villageois désire aussi l'épouser, les garçons du village s'assemblent le soir précédant la noce [...] équipés de sifflets, de clarinettes, d'outils en tôle, de pétrins, de couvercles [...] et, sur un signe donné, font sonner cette musique la plus affreuse qui soit, cela avec une intensité telle qu'on l'entend, par une nuit calme, sur une grande distance $[\ldots]\rangle^{10}$.

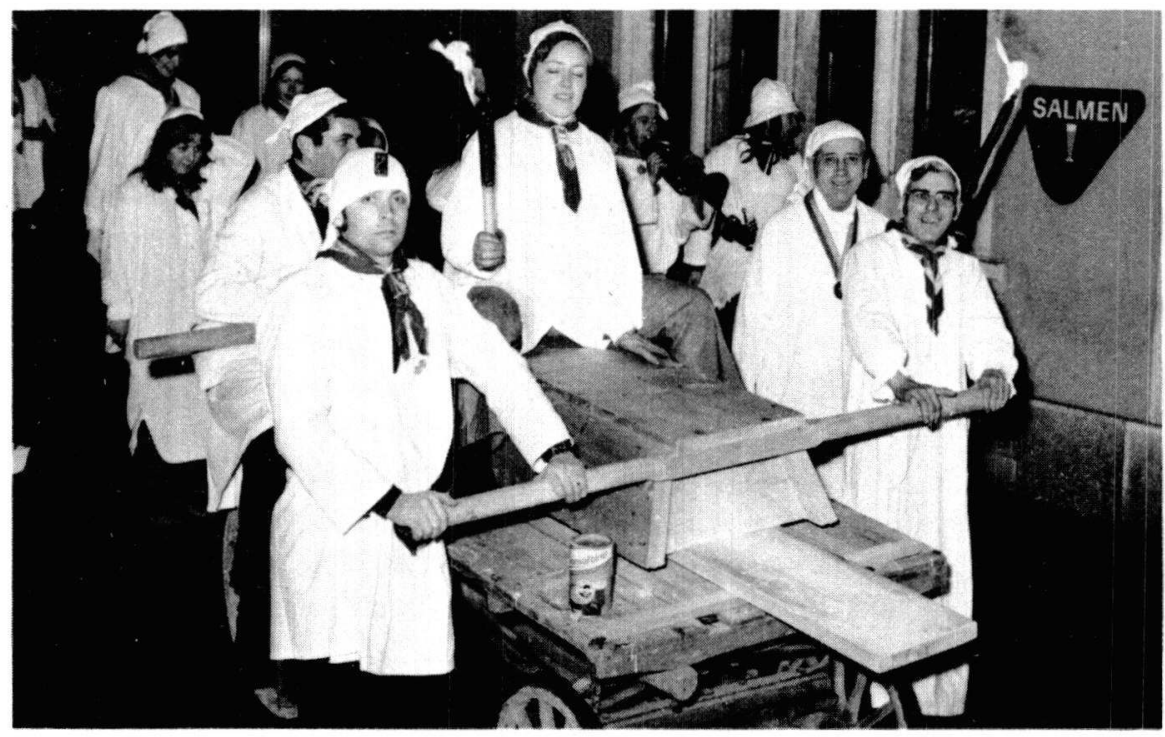

Fig. 4: Le pétrin, servant d'idiophone raclé. Carnaval de Soleure, 1974. Photo: Heri.

9 Chesslete: terme désignant le charivari local.

10 Dans Aus Berg und Thal, II, Glarus, 1985, p. 145. 
C'est par une Mueltschaft ( = «charivari») que les jeunes gens se moquaient d'un veuf qui se mariait pour la seconde fois, comme cela arriva encore en 1972 à Tafers (Fribourg). Dans les années soixante, le pétrin fut frotté à Ursenbach (Berne) aux dépens d'un aubergiste impopulaire, et à Rütschelen (Berne), d'un agent de police détesté. Ces dernières années, le Mueltechratze («frottement du pétrin») ${ }^{11}$ ne s'adressait plus, dans le Brisgau, qu'aux jeunes mariés qui refusaient d'offrir aux célibataires la verrée coutumière. Selon un informateur de Schüpfen (Berne), le frottement du pétrin produit un «hurlement diabolique» ("hornet wie dr Tüfu»), et ce vacarme, affirme un autre informateur à Ursenbach (Berne), peut s'entendre à une distance de huit kilomètres.

Tant que les paysannes feront leur propre pain, le pétrin ne perdra pas sa fonction primaire. De même, comme expression d'une justice populaire profondément enracinée et comme instrument carnavalesque délibérément laid, il ne risque pas non plus de perdre sa fonction secondaire, car le désir de la parodie musicale, du contraste et de l'opposition, en bref, le goût pour les contreinstruments semble être largement répandu. En 1909, dans un essai sur la communauté carnavalesque (Narrengemeinde) d'Appenzell, Alfred Tobler a décrit comment, le lendemain de la Landsgemeinde appenzelloise, on aurait fait le bal non pas au son d'instruments à corde - tels violon, violoncelle, contrebasse et Hackbrett (tympanon) - mais au moyen d'une harmonica et d'un peigne recouvert de papier de soie, afin de ridiculiser les élections et les votations autant que les rituels qui les entourent.

Or, c'est seulement à cette occasion que le peigne recouvert de papier de soie apparaît, dans la tradition populaire suisse, comme un contre-instrument, car il s'agit avant tout d'un instrument de substitution.

\section{Les instruments de substitution}

Le peigne, recouvert de papier de soie ou de papier d'aluminium - voire même, comme à Wartau (Saint-Gall), de la feuille d'un épi de maïs - semble être connu depuis longtemps en Suisse. La Strählmusig ${ }^{12}$ est représentée pour la première fois sur un calendrier zurichois de 1856. Deux soeurs célibataires d'Entschwil dans l'Oberland bernois se souviennent que, voici plus d'un demisiècle, elles s'exerçaient sur leurs Strählflöten («flûtes de peigne») pour exécuter des chants de Noël à deux voix, car, comme d'autres femmes et jeunes filles, elles n'avaient pas les moyens de s'acheter un instrument de musique, si modeste fût-il. Le peigne était, non seulement l'harmonica du pauvre, mais il servait encore d'instrument de danse quand aucun autre instrument n'était disponible, comme l'a décrit Senn-Barbieux (1870: 200) à propos du Toggenbourg:

\footnotetext{
11 Schweizerisches Idiotikon, vol. 3, colonne 930.

12 Strählmusig: «musique de peigne». Cf. Schweizerisches Idiotikon, vol. 5, colonnes 142 et 1077.
} 
«Là alternent toutes sortes de jeux avec yodel, chants et danses, et si l'on ne peut mettre la main sur un instrument de musique, on se débrouille avec le Strählblasen, en soufflant sur un peigne à dents fines enrobé de papier».

Jusque dans les années 1970, à Siebnen (Schwyz) et dans le Muotatal (Schwyz), les garçons qui reculaient devant la difficulté de jouer de la feuille, ou encore de vieux joueurs de guimbarde qui, ayant perdu toutes leurs dents, ne parvenaient plus à tenir l'instrument dans la bouche, recouraient au jeu rudimentaire du peigne. Enfin, le peigne revêt de l'importance pour tous les amateurs de musique qui ne semblent pas avoir un grand talent musical ou qui n'ont jamais eu la possibilité d'apprendre à jouer d'un instrument, mais qui veulent être de la partie quand on s'amuse bien.

Cette fonction du jeu du peigne est illustrée par le petit carnaval organisé en 1974 par l'ensemble de cistres (littéralement: "cithares à manche», «Halszithern») d'Ebnat-Kappel (Saint-Gall), non pas pour un public mais pour son propre plaisir. Par ce charivari, les membres de ce groupe folklorique, qui est surtout composé de femmes et se voue à maintenir la tradition du cistre du Toggenbourg, tenaient à faire participer leurs maris, pour la plupart des agriculteurs ignorant le jeu d'un instrument de musique. Sur l'accompagnement du cistre populaire, les femmes jouaient la mélodie à l'harmonica et au peigne recouvert de papier de soie, tandis que les hommes assuraient la section rythmique en entrechoquant des couvercles, en frappant sur un chaudron avec un balai, en raclant une planche à laver recouverte de tôle ondulée, en agitant des bouteilles vides transformées en hochets au moyen de cuillers à café qui y avaient été introduites, voire en frottant des verres à pied et en jouant du manche à balai ${ }^{13 \bullet}$ (fig. 5).

C'est surtout en Suisse centrale que l'on recourt aux ustensiles de ménage en guise d'instruments d'accompagnement lorsque quelqu'un se met à jouer d'un instrument mélodique - le plus souvent un accordéon - et que les autres ont envie d'y participer sans disposer pour autant d'un instrument de musique conventionnel. Cela peut arriver dans une auberge, en camp de ski, en excursion d'école, lors d'une fête d'entreprise, voire même au service militaire. Selon les cas, ces formations spontanément constituées peuvent atteindre la taille de l'orchestre carnavalesque mentionné plus haut. Mais le plus souvent, on se borne aux instruments suivants: une paire de cuillers, un manche à balai porté sur l'épaule et frappé devant et derrière la tête du joueur à l'aide d'une brosse à farine, d'un Härdöpfustünggü (pilon) ou simplement d'un bâton, enfin, une sorte de contrebasse de fortune.

Pour imiter la contrebasse, le joueur frotte avec ses doigts mouillés, généralement le pouce, une porte ou un battant d'armoire, de préférence en bois de

13 Cette musique carnavalesque figure sous le titre «Vaters Polka» sur un disque - épuisé intitulé Maultrommel-Mundharmonika-Kamm, ex libris EL 12355. 


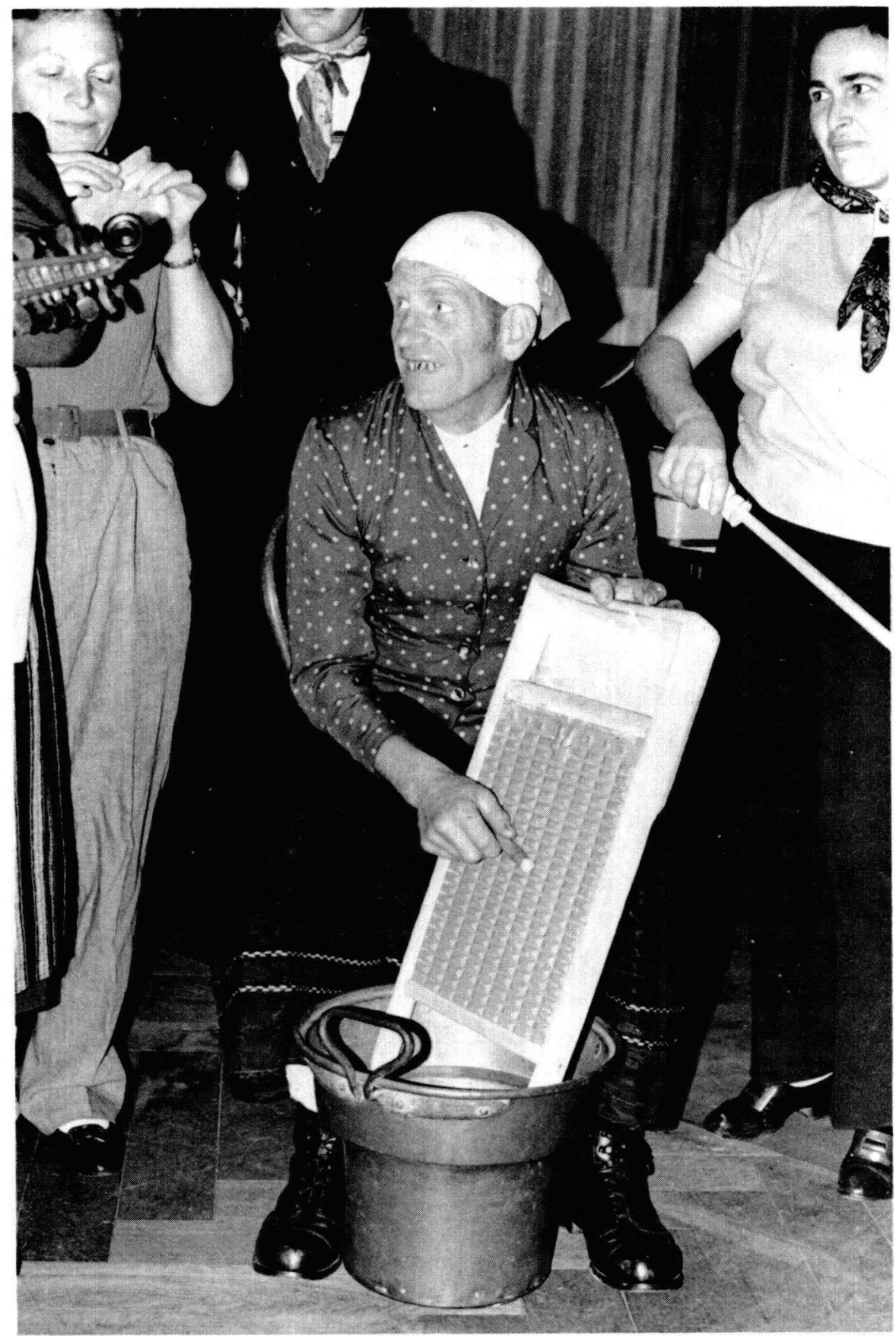

Fig. 5: Joueur de planche à laver recouverte de tôle, lors du carnaval d'Ebnat-Kappel (Saint-Gall), 1974. Photo: B. Bachmann-Geiser. 
sapin, voire même le long d'un mur lambrissé (fig. 6). Pour amplifier le son, on peut utiliser une boille à lait, une caisse ou simplement la valise en carton dans laquelle on transporte l'accordéon schwyzois. Le même effet est obtenu en frottant le siège d'une chaise en bois avec les doigts (fig. 7), ou en poussant le pied d'une chaise sur le parquet. Cette dernière possibilité a été décrite par Hanns In der Gand (1937: 83):

«Dans les casernes aux postes-frontière, on entend parfois l'accordéon accompagné d'un semblant de contrebasse produit au moyen du pied d'une chaise raclant à coups brefs et tranchants sur le plancher».

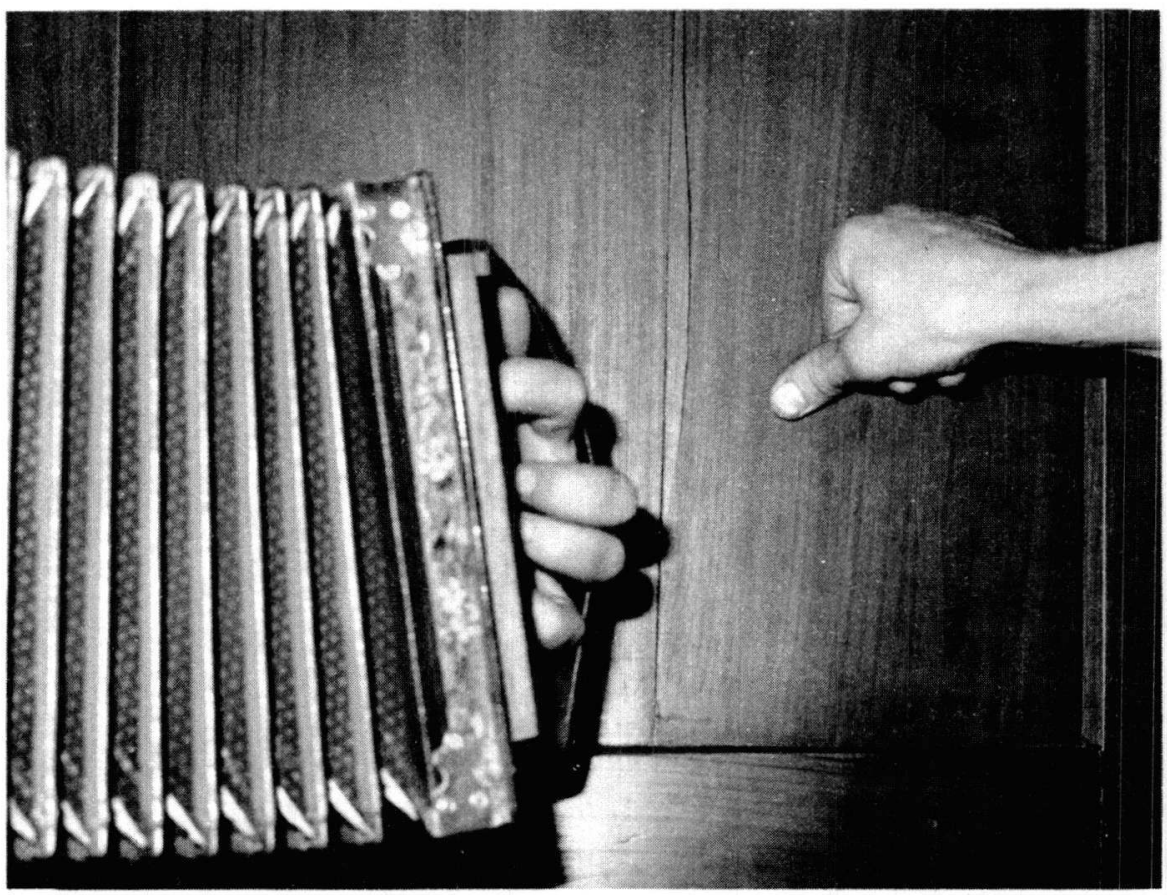

Fig. 6: Frottement du pouce pour produire une basse de substitution. Bettenhausen (Berne), 1974. Photo: B. Bachmann-Geiser.

Le frottement du pouce contre un mur en bois peut être amplifié au moyen d'une bouteille ou d'une douille que l'on tient avec les quatre autres doigts au creux de la main. Une coutume largement répandue consiste à «faire la basse» («Bassmache») à l'aide d'un bâton, d'un outil, d'un manche à balai ou d'une règle. Dans ce cas, on étend l'index mouillé de la main gauche sur le bord d'une table en bois et on le frotte avec le bâton tenu dans la main droite, ce qui provoque la vibration du plateau de la table. On parvient même à varier la hauteur des sons en modifiant la pression exercée sur le doigt ou les points de frottement. 

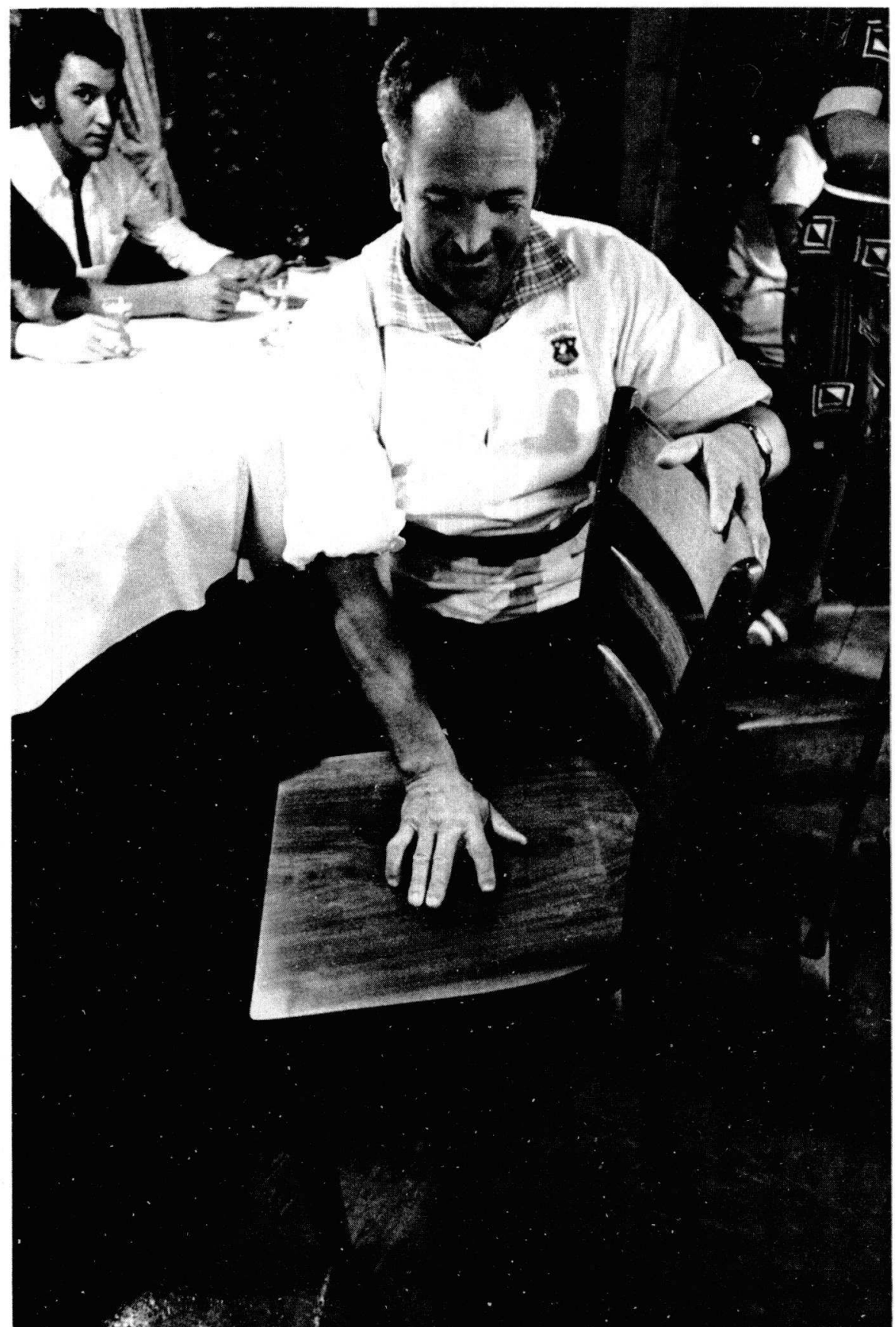

Fig. 7: «Faire la basse» sur une chaise en bois. Bauermusik du Jodelclub de Brunnen (Schwyz), 1976. Photo: K. Wyss. 
Deux tons différents peuvent être obtenus au moyen de deux bouteilles remplies d'eau à différente hauteur. On ignore l'origine du «piano à bouteilles», constitué de bouteilles plus ou moins remplies de façon à produire la gamme et frappées avec un bâtonnet. Le plus ancien témoignage connu à ce sujet est une description, assortie d'une illustration, parue dans la Schweizer MusikerRevue (vol. 10(2/3), 1934). L'existence des «bouteilles musicales», en tant que substitut du piano ou de l'accordéon, est attestée en Valais et en Suisse centrale. Bruno Bürgler, né en 1960 à Ibach (Schwyz), passait pour être un enfant prodige de la musique populaire; il est encore de nos jours un «bouteilliste» (Fläschler) réputé pour son jeu sur dix bouteilles (fig. 8$)^{14}$.

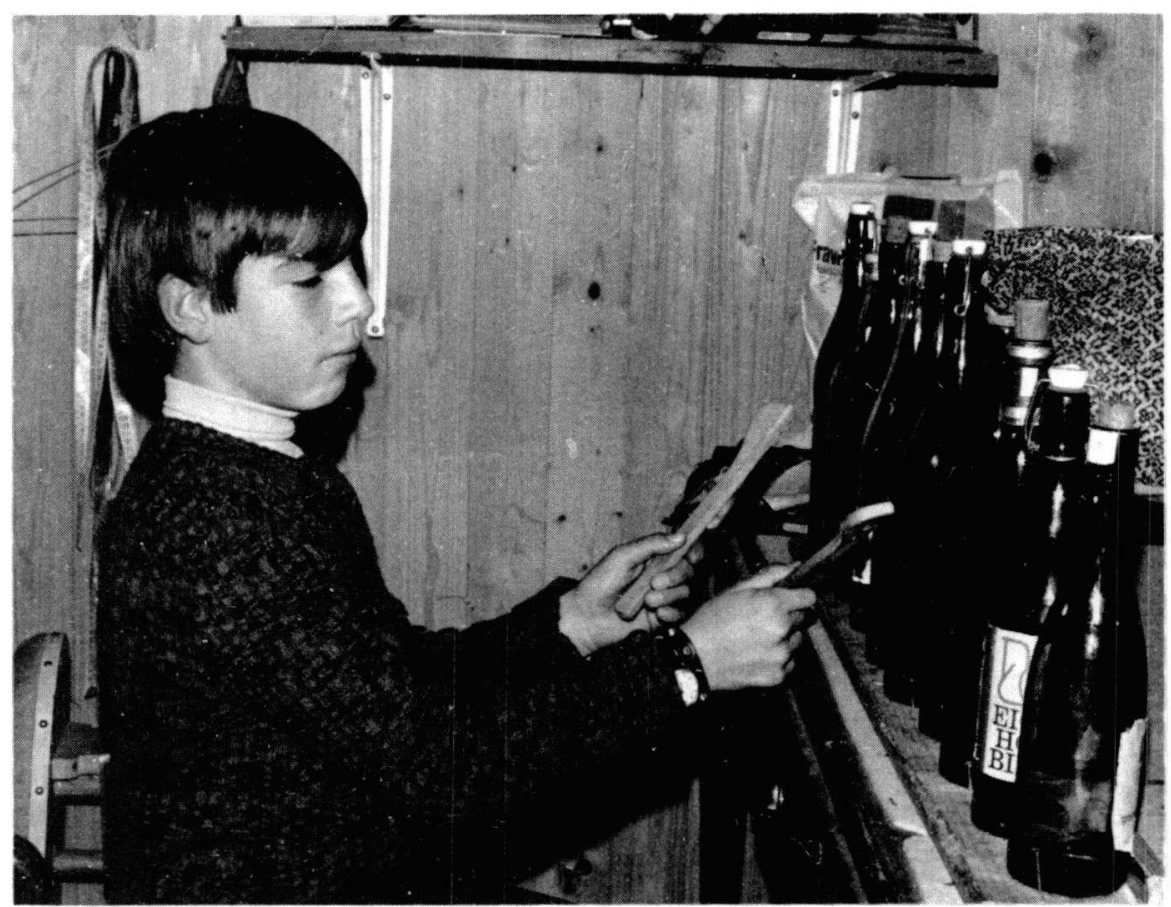

Fig. 8: Bruno Bürgler jouant des bouteilles musicales, Ibach (Schwyz), 1972. Photo: B. Bach mann-Geiser.

L'instrumentarium domestique, auquel recourent volontiers les musiciens de tradition populaire, a été revalorisé par la skifflemusic et le mouvement folk.

Déjà dans les années 1930, les Noirs américains battaient le rythme au moyen de teaspoons et de washboards. Dans les années 1950, cette pratique

14 Pour un exemple du jeu des bouteilles musicales, voir l'anthologie de disques Die Volksmusikinstrumente in der Schweiz, Claves 8012013. 
s'est propagée en Angleterre: en 1956, on dénombrait à Londres plus de six cents ensembles de skiffle (Siniveer 1981: 243), qui ont exercé à leur tour une influence sur la musique populaire et récréative de Suisse. Divers skifflers suisses, comme les Sunday Skifflers, le Wöschbrättbänd ${ }^{15}$ et surtout Pfurri, Gorps und Knirri ont remporté un grand succès avec des instruments domestiques de toutes sortes. Entre 1975 et 1980, les célèbres Argauer Musikanten (qui sont entre-temps retournés à la musique rock) se servaient des instruments domestiques suivants: arrosoir, loquet, bouteille vide, chaînes à neige, harasse, planche à laver, sac à poubelle, cendrier, tire-bouchon, casse-noix, fouet à crème, râpe à noisettes et cuillers; leur disque Leiff de 1976 a même été classé dans le hit-parade.

En réaction contre les coûteux instruments de rock, les instruments domestiques ont également fait leur apparition dans la musique folk ${ }^{16}$. En effet, lorsqu'on écoute les enregistrements des neuf festivals folk organisés entre 1972 et 1980 à Lenzbourg ${ }^{17}$, on constate, notamment sur les enregistrements datant du début des années 1970, l'utilisation de cuillers, appelées spoons par les interprètes; dès 1976, en revanche, on remarque la prépondérance de toutes sortes de cordophones construits sur le modèle d'instruments de musique populaire anglo-saxonne et suisse. En 1974, on a pu entendre à Lenzbourg, à côté d'autres formations populaires suisses, la Rigi-Hundsbuchmusik (fig. 9) de Vitznau (Schwyz), qui, selon la bonne vieille tradition, sifflait avec des feuilles de lierre et jouait de l'harmonica, de la guimbarde, des claquettes (chlefeli), des cuillers et du manche à balai: les amateurs de folk n'ont pas caché leur étonnement face à ces spoons indigènes ${ }^{18}$.

Le jeu des cuillers (fig. 10), attesté en Suisse depuis $1798^{19}$, a pu être observé dans les années 1970 dans la région du lac des Quatre-Cantons, dans le canton d'Obwald, le Meiringen avoisinant, et en particulier dans le canton de Schwyz et dans certaines régions du canton de Glaris, de l'Oberland zurichois et du canton du Valais. Jusqu'en 1975 environ, il était produit presque exclusivement par des cuillers à soupe en métal. Au bistrot, lorsque quelqu'un jouait de l'accordéon et que le rythme en devenait irrésistible, les clients réclamaient souvent une paire de cuillers au personnel. La technique de ce jeu rythmique improvisé, toujours observée de nos jours, est la suivante: le joueur, assis, tient

15 Cf. le disque D'Wöschbrättbänd und Trio Lüönd, Zyt 256.

16 Cf. à ce sujet Hostettler (1981), Hangartner (1981) et Burckhardt-Seebass (1987).

17 Les enregistrements inventoriés des neuf festivals folk sont conservés aux Archives suisses de la chanson populaire, à Bâle.

18 Cf. le disque Rund um die Rigi, EMI Columbia 3 E 062-33856.

19 Cf. Johann-Gottfried Ebel (1798: 172): «J'ai aussi écouté deux garçons chanter des chansons; ils s'accompagnaient en faisant s'entrechoquer deux cuillers en bois qu'ils tenaient entre les doigts et qu'ils agitaient à la manière des Espagnols jouant des castagnettes» (concerne Rhodes intérieures). 


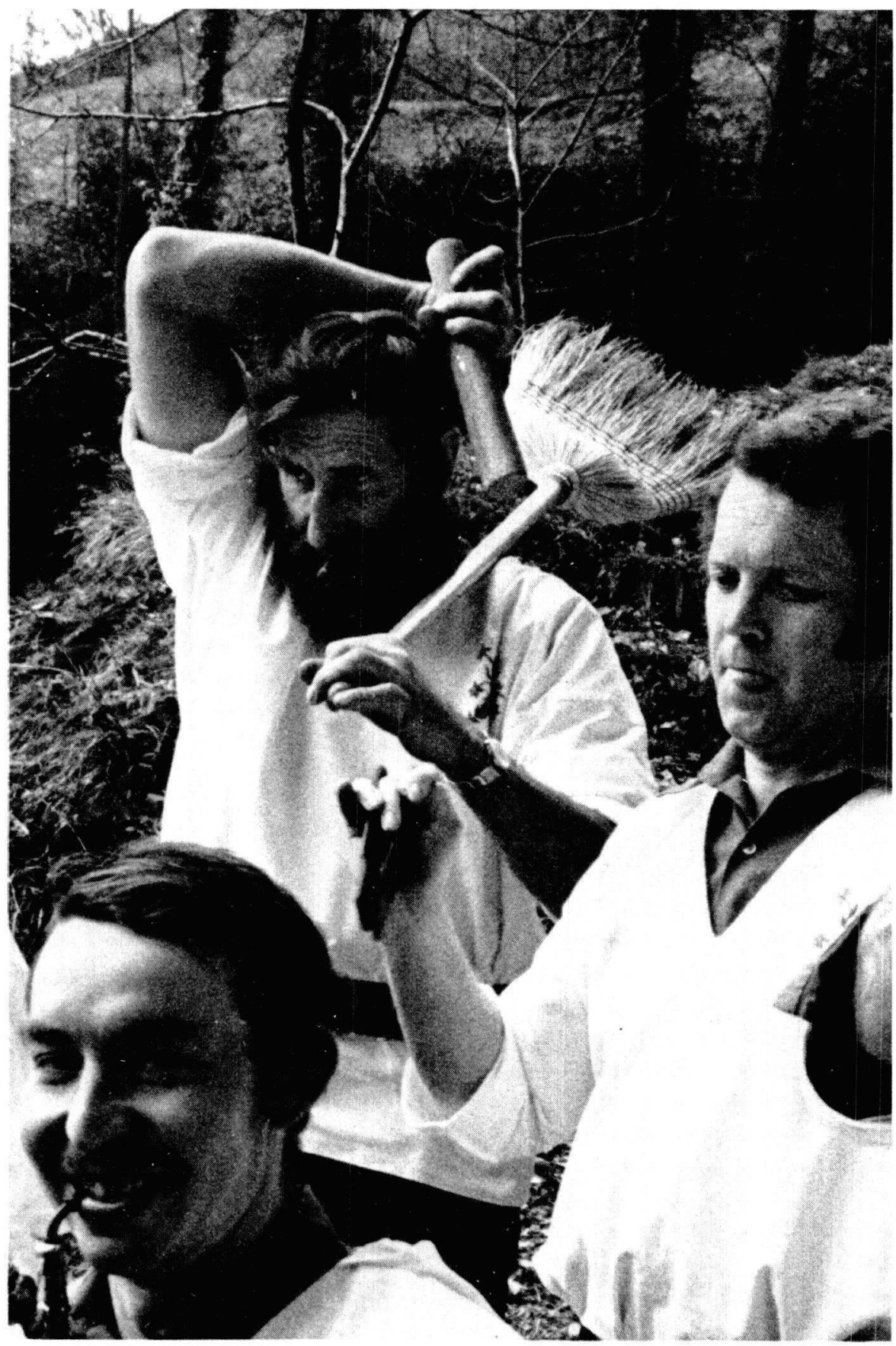

Fig. 9: Joueurs de manche à balai et de cuillers de la Rigi-Hundsbuchmusik, Vitznau (Schwyz), 1975. Photo: SVZ. 
d'une main la paire de cuillers par les manches et la frappe sur l'autre avantbras, les cuisses et la main libre, plus rarement sur les mollets, la poitrine et la tête (fig. 11). Dans les régions où l'on a coutume de boire le café arrosé, notamment en Suisse centrale, on se sert également de cuillers à café. Dans ce cas, nettement moins fréquent que le jeu avec les cuillers à soupe, la position de jeu reste la même, mais la technique est différente: avec la paire de cuillers, on frotte les doigts écartés de la main libre.

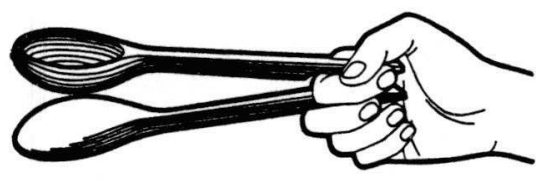

Fig. 10

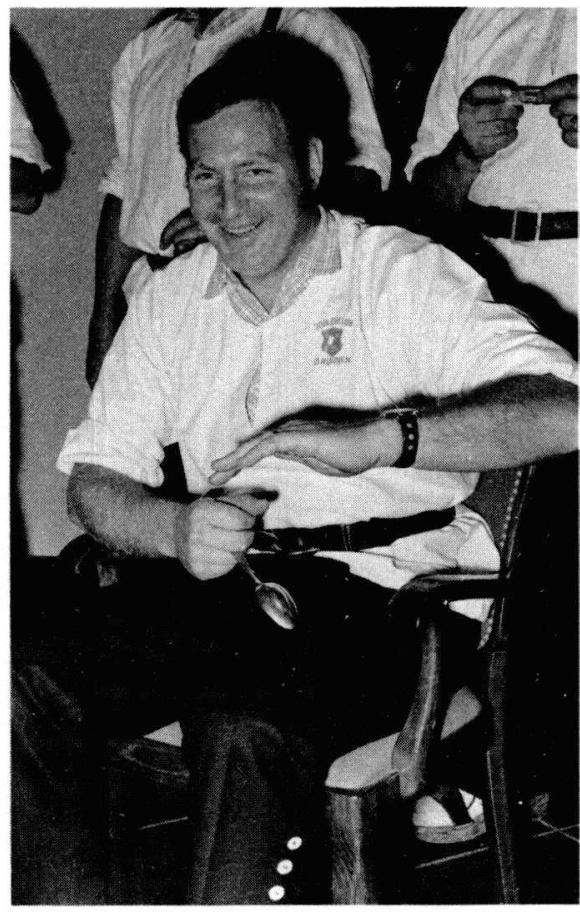

Fig. 11

Fig. 11: Joueur de cuillers de la Bauermusik de Brunnen (Schwyz), 1974. Photo: B. BachmannGeiser.

Si les cuillers proviennent traditionnellement du couvert de table, on a vu apparaître plus récemment le jeu avec des couverts à salade en bois. C'est en avril 1975 à Thalwil (Zurich) qu'on a vu pour la première fois de jeunes citadines venues à une fête de Laendler munies de leurs propres couverts. Probablement sous l'influence des skifflers suisses et du mouvement folk, le jeu des cuillers a connu un nouvel essor. Un informateur de Cham (Zoug) nous a raconté qu'un seul joueur d'accordéon est parfois accompagné par huit joueurs de cuillers, ce qui est considéré comme un véritable honneur pour le musicien. De même, les ensembles populaires comprenant des joueurs permanents de cuillers remportent un succès considérable. Chaque lundi d'été, la Bauermusik 
( = «orchestre champêtre») du Jodelclub de Brunnen (Schwyz) anime par exemple des soirées folkloriques pour touristes étrangers, en jouant d'instruments domestiques jadis méprisés, tel le peigne recouvert de papier de soie, le manche à balai et la «basse sur caisse», ainsi que d'instruments populaires comme l'harmonica et la guimbarde (fig. 12) ${ }^{20}$. Les membres de cette formation "champêtre» sont tout sauf agriculteur; ils auraient donc sans doute les moyens de se procurer des instruments plus sophistiqués. Le recours aux instruments domestiques n'est donc plus dicté chez eux par un manque d'argent, mais plutôt par le désir d'en gagner et d'avoir du succès.

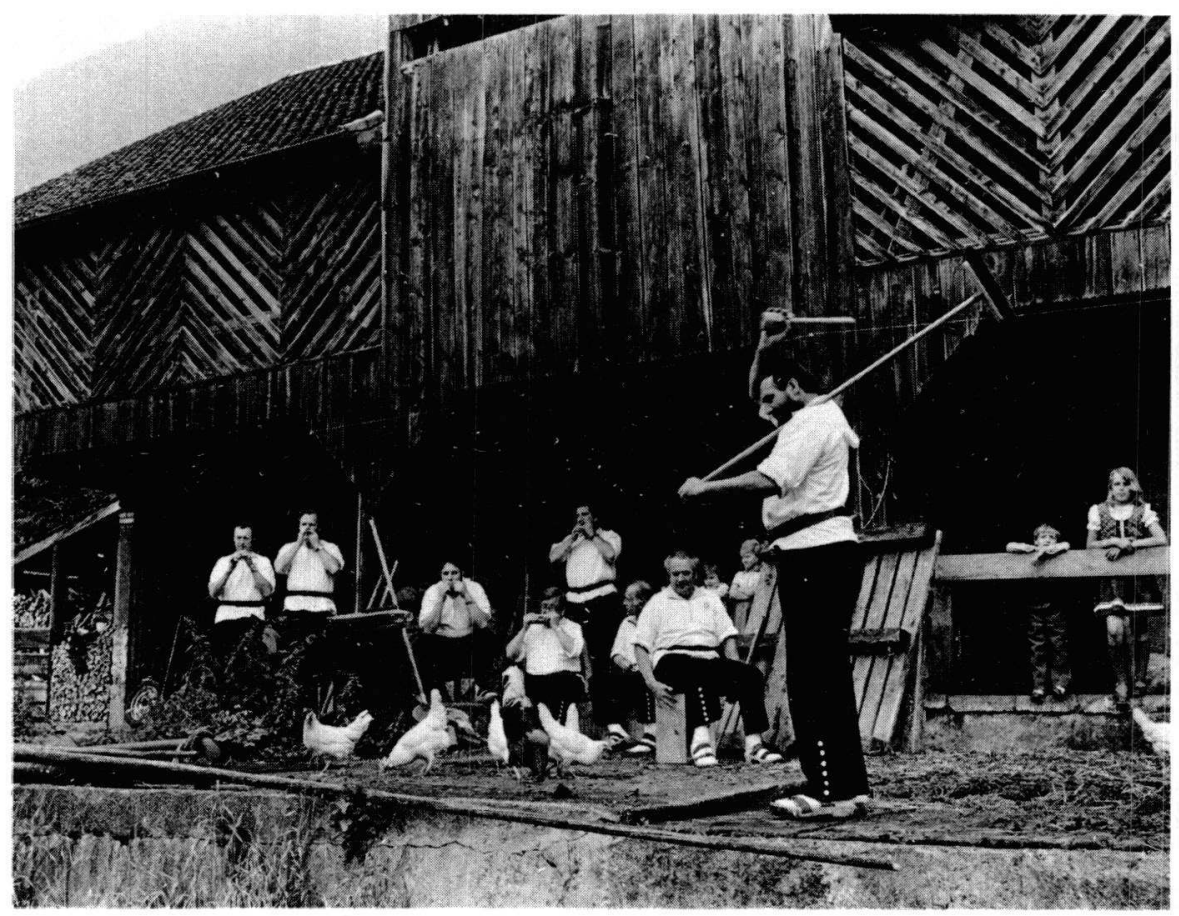

Fig. 12: La Bauermusik du Jodelclub de Brunnen (Schwyz). De gauche à droite: flûte à bec, harmonica, peigne, harmonica, guimbarde, cuillers, «basse sur caisse», manche à balai. Photo: D. Gignoux.

L'essor du jeu des cuillers ces dix dernières années peut être retracé dans la presse, en suivant les chroniques du concours de joueurs de cuillers (Löfflerwettstreit) organisé chaque automne depuis 1977 à Giswil (Obwald) ${ }^{21}$. Si, dans

20 Un exemple musical de cette Bauermusik figure dans l'anthologie de disques Die Volksmusikinstrumente in der Schweiz, Claves 8012/13.

21 Nous tenons à remercier M. Arnold Burch, responsable de la Société de musique de Giswil qui organise du concours des joueurs des cuillers, pour avoir rassemblé le matériel d'archives. 
les années 1970, la plupart des candidats se servaient encore de cuillers à soupe en métal et de couverts à salade, l'utilisation jusque-là sporadique de cuillers en bois sculptées à cette fin était devenue la norme le 30 août 1986. Ainsi le modeste instrument domestique est-il devenu en Suisse un instrument de percussion à la mode qu'on peut se procurer dans tous les grands magasins de musique. Et chaque joueur de cuillers qui se respecte se fait faire son instrument sur commande auprès d'un sculpteur spécialisé.

Depuis plus de dix ans, un sculpteur talentueux, Franz Dillier d'Engelberg (Obwald), rénommé pour ses figurines de crèche, sculpte dans du bois de hêtre ou de cerisier des cuillers en forme de cuillers de cuisine d'une longueur de 40 $\mathrm{cm}$, munies d'encoches pour les doigts ${ }^{22}$. Quant à Werner Reichmuth de Cham (Zoug), ouvrier à la retraite, il a commencé à sculpter des cuillers par passion pour la musique de Laendler et de l'Älplerschlagzeug («batterie des pâtres»). Par comparaison avec les instruments de Dillier, légers et munis de sculptures en encoche bien travaillées et lissées, l'ouvrage de ce dernier est plus lourd, quelque peu grossier et anguleux, mais il s'attache à choisir du bois bien veiné pour la fabrication de cuillers destinées à la décoration murale. Reichmuth choisit son bois avec soin (hêtre, prunier et noisetier, mais aussi pommier et chêne), sachant que seuls les bois bien secs provenant d'arbres en bonne santé produisent le son tant apprécié par ses clients; il regrette que seuls de vieux pruniers noueux puissent être abattus, car les cuillers faits dans ce bois sont très recherchées. De plus en plus, Reichmuth fabrique aussi des cuillers de dimensions plus réduites pour les enfants. Ainsi, l'instrument jadis réservé aux hommes fréquentant les auberges est maintenant accessible aux femmes et aux jeunes filles ${ }^{23}$. Pour sa part, Beat Kollegger d'Alvaneu (Grisons), un jeune musicien et facteur de divers instruments de musique populaire, orne ses cuillers du blason grison ou helvétique et ne peut faire face à la demande croissante qu'en mettant à contribution un atelier d'handicapés.

Qui sont les joueurs de ces centaines, voire de ces milliers de paires de cuillers? Comment ce modeste ustensile domestique s'est-il transformé en un instrument de percussion fabriqué par des spécialistes et mis à l'épreuve lors de concours? Faute d'enquêtes en la matière, on ne peut répondre à ces questions qu'en émettant quelques observations et hypothèses.

Depuis les années 1970, le jeu des cuillers, tout comme celui d'autres instruments populaires, a connu un nouvel essor grâce à la renaissance de la musique populaire. Même fabriquées sur commande, les cuillers demeurent bon marché; leur maniement s'apprenant en outre facilement en autodidacte, c'est un instrument accessible à quiconque veut bien le pratiquer.

D'autre part, on ne peut jouer des cuillers qu'en accompagnement d'un instrument mélodique, de préférence un accordéon. A l'époque, il fallait d'abord un joueur d'accordéon schwyzois pour donner envie de battre le rythme.

22 Pour une illustration, voir Bachmann-Geiser (1981: 13).

23 Lors du concours des Löffler de 1986, une fillette de dix ans, Sereina Kamm de Hinwil (Lucerne) l'emporta sur ses collègues masculins. Cf. Vaterland, ler septembre 1986. 
Aujourd'hui, c'est l'inverse: on se procure des cuillers ayant une bonne sonorité pour s'exercer en accompagnant un disque ou une cassette. D'une manière générale, les joueurs de cuillers préfèrent des interprètes virtuoses de la musique d'accordéon et de clarinette, et des danses rapides comme le Laendler et le Schottische.

Les joueurs de cuillers anonymes qui exhibent leur art lors de concerts de Laendler (ce qui ne plaît pas toujours aux autres auditeurs) ont du plaisir à pratiquer leur instrument en situation. Mais on rencontre aussi les amateurs de danse qui, contraints par le statut de «spectateurs», tentent de s'y soustraire en participant activement à la musique en restant assis sur leur siège. Considéré sous cet angle, le nouvel essor du jeu des cuillers n'est pas seulement la manifestation bienvenue d'une musique de type «je- $k a-m i\rangle^{24}$, mais encore une des conséquences de la commercialisation toujours plus avancée de la musique populaire suisse.

\section{Bibliographie}

\section{BACHMANN-GEISER Brigitte}

1981 Die Volksmusikinstrumente der Schweiz. Leipzig: VEB Deutscher Verlag für Musik; Zürich: Atlantis. [Handbuch der europäischen Volksmusikinstrumente, Serie I, Bd. 4].

1984 «Der Zürcher Schulsilvester». 168. Neujahrsblatt der Allgemeinen Musikgesellschaft Zürich auf das Jahr 1984. Zürich: Kommissionsverlag Hug.

BIELAWSKI Ludvik

1985 «Brummtopf und Brummbass in deutscher und polnischer Volkstradition». Studia instrumentorum musicae popularis 8: 108-11.

BURCKHARDT-SEEBASS Christine

1987 «'Gang, hol d'Gitarre'. Das Folkfestival auf der Lenzburg, 1972-1980 und die Schweizerische Folkbewegung». Schweizerisches Archiv für Volkskunde 83(3-4): 154-168.

EBEL Johann-Gottfried

1798 Schilderung der Gebirgsvölker der Schweiz. 1. Bd. Leipzig.

ELSCHEK Oskár

1983 Die Volksmusikinstrumente der Tschechoslowakei, Teil 2. Die slowakischen Volksmusikinstrumente. Leipzig: VEB Deutscher Verlag für Musik. [Handbuch der europäischen Volksmusikinstrumente, Serie 1, Bd. 2].

HANGARTNER Urs

1981 «Volk mit 'F'. Anmerkungen zur sogenannten Folkbewegung». Schweizer Volkskunde 71: 78-82.

HERRMANN Hildegard

1978 «Kinderinstrumente. Versuch einer Bestimmung aus volksmusikinstrumentenkundlicher Sicht». In W. Deutsch und M. Schneider, Beiträge zur Volksmusik in Tirol. Innsbruck.

HOSTETTLER UrS

1981 «Über die Schweizerische Folkbewegung». Schweizer Volkskunde 71: 73-77.

24 «Je-ka-mi» = Jeder kann mitmachen, «chacun peut participer». 
IN DER GAND Hanns

1937 «Volkstümliche Musikinstrumente der Schweiz». Schweizerisches Archiv für Volkskunde 36: 73-120.

JAKOB F. \& BRINER A.

1961 «Das Musikbild und die Hausorgel im Landgut zur 'Schipf' in Herrliberg-Zürich», $n$ : 145. Neujahrsblatt der Allgemeinen Musikgesellschaft auf das Jahr 1961.

KUMER Zmaga

1986 Die Volksmusikinstrumente in Slowenien. Leipzig: VEB Deutscher Verlag für Musik. [Handbuch der europäischen Volksmusikinstrumente, Serie I, Bd. 5].

KUNZ Ludvik

1974 Die Volksmusikinstrumente der Tchechoslowakei. Leipzig: VEB Deutscher Verlag für Musik. [Handbuch der europäischen Volksmusikinstrumente, Serie I, Bd. 2].

SACHS Curt

1926 «Heidenlärm und Kinderspiel». Velhahgen und Klasings Monatshefte 41, 1. H.S.: 93-97.

SÁROSI Bálint

1967 Die Volksmusikinstrumente Ungarns. Leipzig: VEB Deutscher Verlag für Musik. [Handbuch der europäischen Volksmusikinstrumente, Serie I, Bd. 4].

SENN-BARBIEUX Walter

1870 Charakterbilder Schweizerischen Landes, Lebens und Strebens. St. Gallen.

SINIVEER Kaarel

1981 Folk-Lexikon. Hamburg: Rowohlt.

TOBLER Alfred

1909 «Appenzeller Narrengemeinde». Heiden.

VIRDUNG Sebastian

1970 Musica getutscht. Éd. en fac-simile par K.W. Niemöller. Basel, Paris, London: Bärenreiter. 